\title{
Etiology of Community-Acquired Pneumonia
}

\section{Requiring Hospital Admission in Adults with and Without Cancers: A Single-Center Retrospective Study in China}

This article was published in the following Dove Press journal:

Infection and Drug Resistance

\author{
Xinxin Wang ${ }^{1, *}$ \\ Haihua Zhang ${ }^{1}$ * \\ Tao Zhang (D) ${ }^{\prime}$ \\ Lei Pan' \\ Ke Dong ${ }^{2}$ \\ Ming Yang ${ }^{2}$ \\ Ruina $\mathrm{Ma}^{\mathrm{l}}$ \\ Yujuan $\mathrm{Li}^{\prime}$ \\ 'Department of Pulmonary and Critical \\ Care Medicine, The Second Affiliated \\ Hospital of Air Force Medical University, \\ Xi'an, People's Republic of China; \\ ${ }^{2}$ Department of Laboratory, The Second \\ Affiliated Hospital of Air Force Medical \\ University, Xi'an, People's Republic of \\ China
}

*These authors contributed equally to this work
Correspondence: Tao Zhang

Department of Pulmonary and Critical

Care Medicine, The Second Affiliated

Hospital of Air Force Medical University,

Xi'an, People's Republic of China

Email zhangft@fmmu.edu.cn
Background: The etiology and distribution of community-acquired pneumonia (CAP) vary periodically and geographically. The clinical evaluation of CAP among patients with cancers remains unknown.

Patients and Methods: This retrospective hospital-based study on adult CAP was conducted in Tang Du Hospital, China, from September 2018 to August 2019. The demographic characteristics, clinical manifestations and laboratory data were extracted from medical records and compared between CAP patients with and without cancers. Univariable and multivariable logistic regression methods were used to explore risk factors associated with CAP patients with and without cancers. Results: Data from 149 CAP patients with cancers and 268 CAP patients without cancers were analyzed. Patients without cancers were more likely to show fever, cough and yellow sputum, higher level of neutrophil count than the cancer patients. Klebsiella pneumoniae (K. pneumoniae $14.77 \%$ vs $9.33 \%, \mathrm{p}=0.093$ ) and Streptococcus pneumoniae (S. pneumoniae $16.11 \%$ vs $11.57 \%$, $\mathrm{p}=0.189)$ were among the most commonly encountered pathogens in both the groups. Pseudomonas aeruginosa (P. pneumoniae $26.50 \%$ vs $11.41 \%, \mathrm{p}<0.001$ ), Mycoplasma pneumoniae (M. pneumoniae $8.21 \%$ vs $1.34 \%, \mathrm{p}=0.003)$, and filamentous fungi $(10.82 \%$ vs $4.7 \%, \mathrm{p}=$ 0.033 ) were predominant in CAP patients without cancers. Haemophilus influenzae (H. influenzae $22.15 \%$ vs $14.18 \%, \mathrm{p}=0.038$ ) and methicillin-resistant Staphylococci (MRS 23.49 vs $15.68, \mathrm{p}=$ 0.049 ) were more prevalent for CAP cancer patients. Certain pathogens were increasing in a cold season. In patients without cancers, MRS, $H$. influenzae and $P$. aeruginosa were associated with central nervous system (CNS) disease, connective tissue disease, bronchiectasis, respectively. In addition, healthy adults were likely to be infected with $M$. pneumoniae showing fever.

Conclusion: CAP patients with cancers had atypical clinical manifestations and showed no distinct increase in inflammatory markers. The predominant pathogens differed as well as similar between the CAP patients with and without cancers. Certain pathogens follow a seasonal pattern. CNS disease, connective tissue disease and bronchiectasis were associated with the predominant pathogens in patients without cancers.

Keywords: community-acquired pneumonia, etiology, pathogen, epidemiology, cancer

\section{Introduction}

Community-acquired pneumonia (CAP) is a common infectious disease worldwide. Pneumonia was the fourth leading cause of death globally and the first in lowincome countries in 2016. ${ }^{1}$ In general, older individuals and patients with 
underlying comorbidities and immunocompromised patients, such as those with cancers, are at a higher risk for CAP. ${ }^{2,3}$ Previous studies have reported that androgen deprivation for prostate cancer was associated with an increased risk of hospitalization for community-acquired pneumonia in men with prostate cancer. ${ }^{4}$ In cancer patients, the incidence rate of pneumonia was increased approximately more than threefold compared to healthy subjects. ${ }^{3}$ In addition, the incidence rate of CAP was high in patients with cancers with strong variations by cancer subtype. Lung cancer patients had a 21-fold higher risk compared to the cohort without cancer; however, breast cancer patients had 1.7-fold. ${ }^{5}$ Advancements in therapeutic protocols (such as operation, radiation, chemotherapy, targeted therapy, and immunotherapy) have led to prolonged survival and a good quality of life. However, these advancements are also associated with some serious adverse effects. The number of cancer patients with CAP requiring hospital admission is constantly increasing. Furthermore, in patients with advanced lung cancer who have had lung resection, CAP is a serious and often lifethreatening condition. ${ }^{6-8}$ The rate of postoperative bacterial pneumonias remains high and may confer an increased risk of metastasis. ${ }^{9}$

During the initial management of a patient with CAP, it is crucial to identify possible risk factors and empirical antimicrobial treatments, as the causative pathogen is unknown. Usually, traditional culture-based diagnostic methods may not be sufficiently sensitive to detect pathogens, as even the more common bacterial pathogens require at least $48 \mathrm{hrs}$ to culture. It has been reported that a microbiological identification was found in about $30 \%$ of hospitalized patients with CAP. ${ }^{10,11}$ Pathogens were often not found even in severe CAP in the intensive care unit. ${ }^{12,13}$ It is often difficult to obtain a good-quality lower respiratory tract specimen for culture, leaving physicians with poor-quality specimens or no culture data at all, particularly among patients with advanced cancer and severe infection. Only a minority of pathogen isolates are available from bronchoalveolar lavage fluid (BALF). ${ }^{14}$

Nevertheless, the etiology of CAP patients with cancers remains limited. The distribution of pathogens in CAP varies periodically and geographically. The reported results were mainly based on traditional culture results, and we hypothesized that the etiology of CAP might differ from that reported previously, as rapid diagnostic tests were introduced extensively.
Thus, the aim of this article is to compare the prevalence, microbiology, and risk factors of CAP in hospitalized patients with and without cancer to help clinicians choose more appropriate management strategies and minimize the unnecessary use of broad-spectrum antibiotics.

\section{Patients and Methods}

\section{Study Design and Site}

This single-center retrospective observational study included adult patients with a diagnosis of CAP, who had been admitted to the Tang Du Hospital in Xi'an, Shaan xi province, between September 2018 and August 2019. The Tang Du Hospital affiliated to the Air Force Medical University is a 3000-bed capacity hospital and is one of the five biggest tertiary care hospitals in the region. The hospital, which sees 2 million outpatients and 130 thousand inpatients annually, mainly serves as the referral center for patients requiring tertiary medical care from Xi'an City and other cities of the Shaan Xi province in the northwest district of China.

\section{Patient Collection}

Patients were recruited for the study based on an initial clinical diagnosis of CAP by the admitting physician, according to the clinical practice guidelines for the diagnosis and management of CAP (Chinese Respiratory Association, 2016). ${ }^{15}$

The inclusion criteria were as follows:

1. Pneumonia developed outside the hospital, including within $48 \mathrm{hrs}$ after admission with the pathogen in the incubation period;

2. There were pneumonia-related clinical manifestations, including

(1) Newly developed cough and expectoration, or aggravation of original respiratory disease symptoms;

(2) Fever;

(3) Signs of lung consolidation and (or) wet rale;

(4) Peripheral blood leukocyte count $>10 \times 10^{9} / \mathrm{L}$ or $<4$ $\times 10^{9} / \mathrm{L}$, with or without a shift to the left;

3. Chest imaging revealed new shadow with patchy infiltrations, leaf or segment consolidation, groundglass shadow, and interstitial change, with or without pleural effusion.

4. Pathogens should be clearly detected in sputum or BALF.

5. Patients aged 18 years or older. 
All the patients meeting 1, 3, 4, 5 and one of the second items were eligible.

The exclusion criteria were as follows:

1. The cause of hospital admission was Any other noninfectious pulmonary interstitial diseases, pulmonary edema, atelectasis, pulmonary embolism, pulmonary eosinophil infiltration, and pulmonary vasculitis, except cancers.

2. Tuberculosis and viral pneumonia,

3. Microbial colonization without therapy.

If the patients met one of the three items, they were excluded.

PSI scores and other medical conditions were used to deem patients with CAP eligible for hospital admission. ${ }^{15}$ For analysis, patients were divided into two groups (one group with cancers and another without cancers) according to the presence of co-cancer.

\section{Data Collection}

Patient information, including demographic characteristics, clinical symptoms and signs, and laboratory data were extracted from medical records. Two researchers then independently reviewed the collected data in cases recorded forms and a third researcher adjudicated any disagreements.

\section{Microbiological Detection}

Causative pathogens were mainly identified from sputum and BALF samples were collected within $24 \mathrm{hrs}$ after hospital admission according to local standard operating procedures. Samples were evaluated when the gram-staining revealed numerous leukocytes leukocytes $(>25$ in a microscopic field at $\times 100$ magnification $)$ and few squamous epithelial cells $(<10$ in a microscopic field at $\times 100$ magnification). The sample size should not be less than $6 \mathrm{~mL}$. Nucleic acid amplification techniques (loop-mediated isothermal amplification, LAMP, CaptialBio Technology, China) and routine microbiological culture (microdilution method) were used to detect pathogens. As for conventional culture, when the sputum contained $\geq 10^{5}$ colony-forming units (CFU)/mL or the BALF showed $\geq 10^{3}$ $\mathrm{CFU} / \mathrm{mL}$ bacteria, the identified strains were then considered as the causative pathogens. A nucleic acid load of at least $5 \times$ $10^{2}$ copies/ $\mu \mathrm{L}$ in LAMP was considered a positive result. Methicillin-resistant Staphylococci(MRS), Mycoplasma pneumoniae(M. pneumoniae), Legionella pneumophila and Chlamydia pneumoniae were detected by LAMP.
Streptococcus pneumoniae (S. pneumoniae), Staphylococcus aureus, Pseudomonas aeruginosa( $P$ aeruginosa), Haemophilus influenzae(H. influenzae), Klebsiella pneumoniae(K. pneumoniae), Escherichia coli, Acinetobacter baumannii, Stenomonas maltophilia were tested by either culture or LAMP. Fungi, actinomyces and other rare bacteria were detected by culture.

\section{Statistical Analysis}

In the statistical analysis, categorical variables showed as counts (percentages) were compared with the chi-square test and the Fisher's exact test. Continuous variables expressed as the median values with non-normal distribution or the means \pm SD for those with normal distribution were compared using the Student's $t$-test or the nonparametric Mann-Whitney $U$-test when appropriate. All tests were two-tailed and the significance level of $\alpha$ was set at 0.05 .

To identify potential risk factors, univariate and multivariable logistic regression were performed to assess the association of demographic and clinical manifestations with some pathogens; odds ratios (OR), and 95\% confidence intervals (CI) were calculated. Variables, that showed a significant result univariately ( $p<0.05$ ), was included in the corresponding multivariate logistic regression by a backward stepwise model. Considering the number of patients of certain pathogens $(2 \leq \mathrm{n} \leq 71)$ in our study and to avoid overfitting in the model, six variables (bronchiectasis, sex, connective tissue disease, CNS diseases, no comorbidities, fever) were chosen for multivariable analysis on the bases of clinical constraints. All analyses were performed with IBM SPSS Statistics V21.0.

\section{Results}

A total of 14,417 adults were diagnosed with pneumonia in our institution from September 2018 to August 2019. Eight thousand eight hundred and eighteen patients had no pathogen findings. Because microbiological detection was not performed in some patients or others had negative bacterial results (Figure 1). Two thousand seven hundred and thirty-six cases and 1752 cases were hospital-acquired pneumonia (HAP) and ventilator-associated pneumonia (VAP), respectively. In addition, 655 patients were diagnosed with non-infectious pneumonia. After the patients with concomitant tuberculosis, and a virus infection were excluded, 417 patients remained, who were included in the analysis. There were 149 CAP patients with cancer (lung cancer up to $73.8 \%$ ) and 268 CAP patients without cancer (Figure 2).

Figures 3, 4 and 5 illustrate the annual trend of the number of cases of pneumonia with different causative 


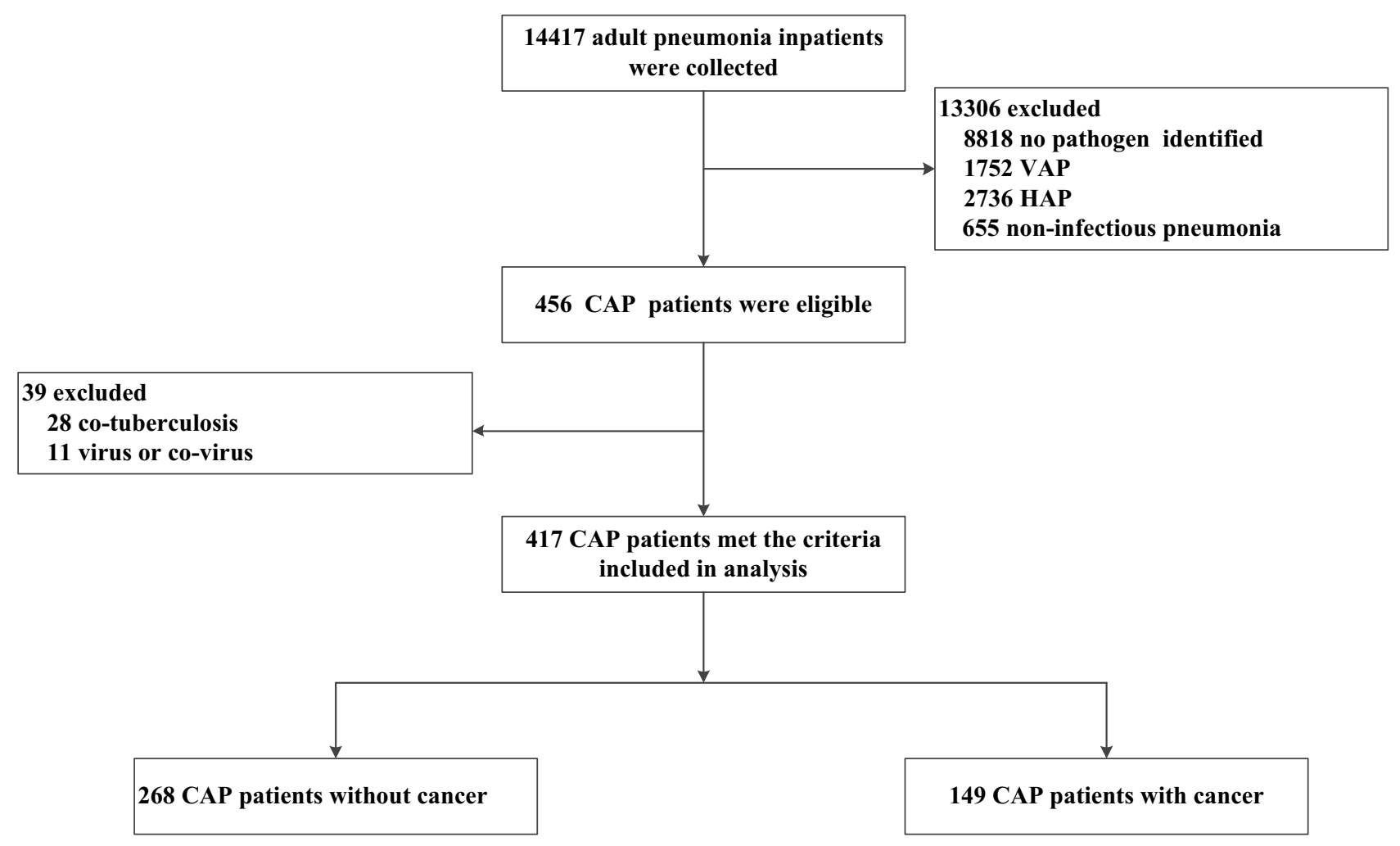

Figure I Flow diagram of patients screening and recruitment.

Abbreviations: CAP, community-acquired pneumonia; HAP, hospital-acquired pneumonia; VAP, ventilator-associated pneumonia.

agents. As seen in Figure 5, patients infected with $P$. aeruginosa without cancer showed sharp peaks in March and September. The circulation of K. pneumoniae and $S$. pneumoniae in cancer patients were increasing in September and October, respectively (Figures 3 and 4,

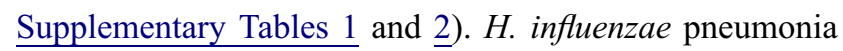

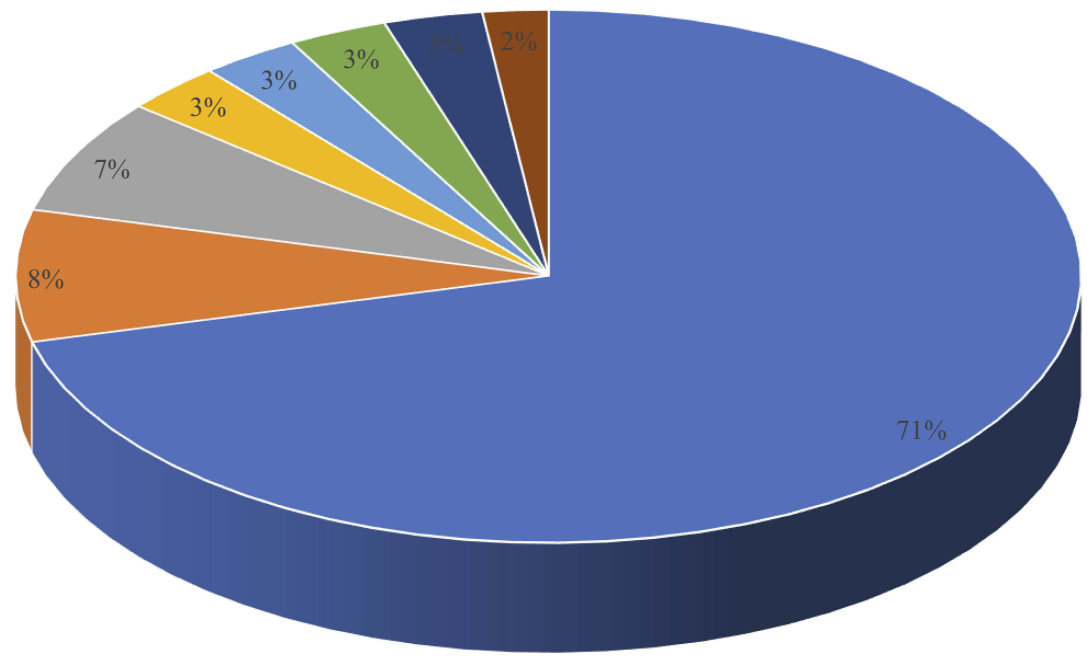

$$
\begin{array}{llll}
\text { - Lung cancer }(\mathrm{n}=105) & \text { - Esophaeal cancer }(\mathrm{n}=12) & \text { " Hematologic } \operatorname{cancer}(\mathrm{n}=11) & \text { - Laryngeal Cancer }(\mathrm{n}=5) \\
\text { - Genital cancer }(\mathrm{n}=5) & \text { " Other } \operatorname{cancers}(\mathrm{n}=5) & \text { - Two cancers }(\mathrm{n}=4) & \text { - Gastric } \operatorname{cancer}(\mathrm{n}=2)
\end{array}
$$

Figure 2 Prevalence of different types of cancers. 


\section{Streptococcus pneumoniae}

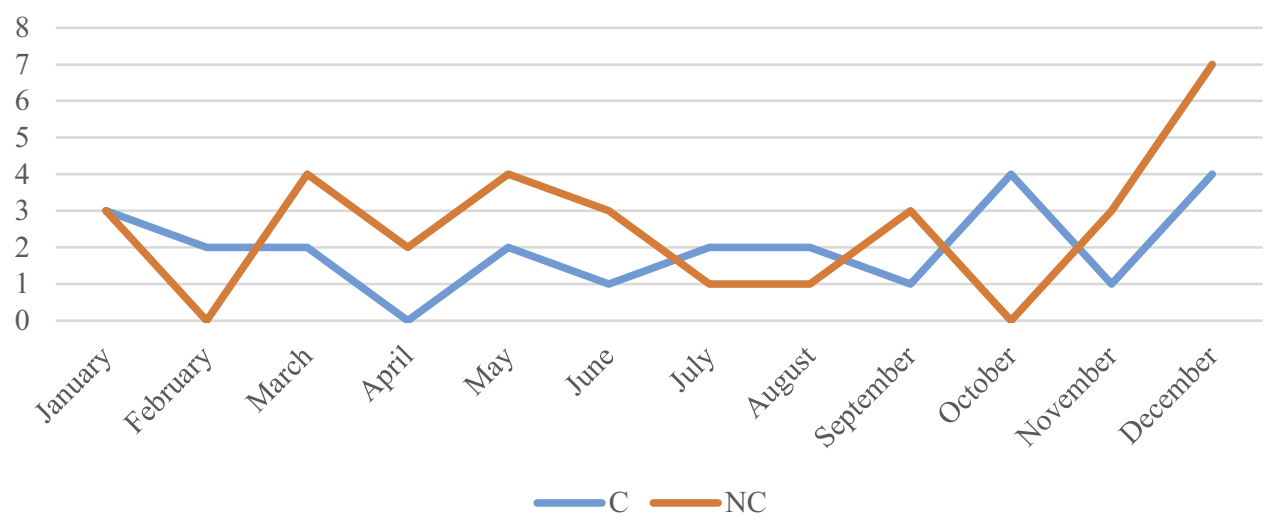

Figure 3 Seasonal distribution of Streptococcus pneumoniae among CAP patients with cancers and without cancers between September 2018 and August 2019. Abbreviations: CAP, community-acquired pneumonia; NC, no cancer; C, cancer.

with the most number of cases occurred in July in both groups (Figure 5, Supplementary Tables 1 and 2).

As shown in Table 1, the median age of the CAP patients without and with cancer were 60 and 61 years, respectively. In addition, cancer patients had a lower frequency of smoking $(72.76 \%$ vs $69.13 \%, p<0.001)$ and fewer other underlying diseases $(26.12 \%$ vs $61.07, \mathrm{p}<$ $0.001)$. Patients without cancer $(73.88 \%)$ had more other chronic airway diseases such as bronchiectasis $(23.51 \%$ vs $3.36 \%, \mathrm{p}<0.001)$, connective tissue disease $(11.19 \%$ vs $5.37 \%, \mathrm{p}=0.048)$, and CNS disease $(15.67 \%$ vs $2.68 \%$, $\mathrm{p}<0.001)$. Patients without cancer were more likely to show fever $(44.40 \%$ vs $22.82 \%, \mathrm{p}<0.001)$ with a higher median body temperature $\left(37^{\circ} \mathrm{C}\right.$ vs $\left.36.8^{\circ} \mathrm{C}, \mathrm{p}<0.001\right)$, respiratory failure $(22.39$ vs $10.74, \mathrm{p}=0.003)$, cough $(87.31 \%$ vs $73.15 \%, \mathrm{p}<0.001)$, and yellow sputum
$(39.55 \%$ vs $28.86 \%, \mathrm{p}=0.029)$. There were no distinct increases in the median value of blood cells between two groups, except the neutrophil counts (5.40 vs 5.01, p = $0.044)$ were higher in patients without cancer. In the event of a microbial infection.

$K$. pneumoniae and $S$. pneumoniae were among the predominant pathogens in both the groups (Table 2). P. aeruginosa $(24.63 \%$ vs $10.07 \%, \mathrm{p}<0.001)$ and M. pneumoniae $(8.21 \%$ vs $1.34 \%, \mathrm{p}<0.004)$ were predominant in patients without cancers. $H$. influenzae $(18.80 \%$ vs $11.20 \%, p=0.032)$ was more prevalent in cancer patients.

Table 3 shows the overall distribution of pathogens as isolated alone and/or in combination with other pathogens (mixed infection). MRS (23.49\% vs $15.68 \%, \mathrm{p}=0.049)$ and filamentous fungi $(10.82 \%$ vs $4.7 \%, p=0.033)$ were the

\section{Klebsiella pneumoniae}

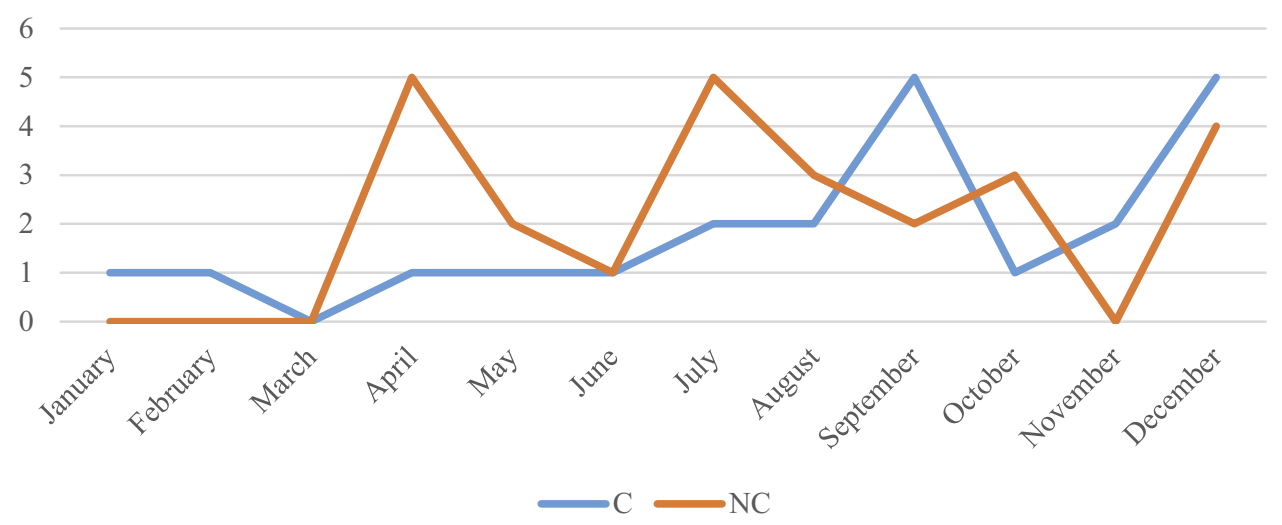

Figure 4 Seasonal distribution of Klebsiella pneumoniae among CAP patients with cancers and without cancers between September 2018 and August 2019. Abbreviations: CAP, community-acquired pneumonia; NC, no cancer; C, cancer. 


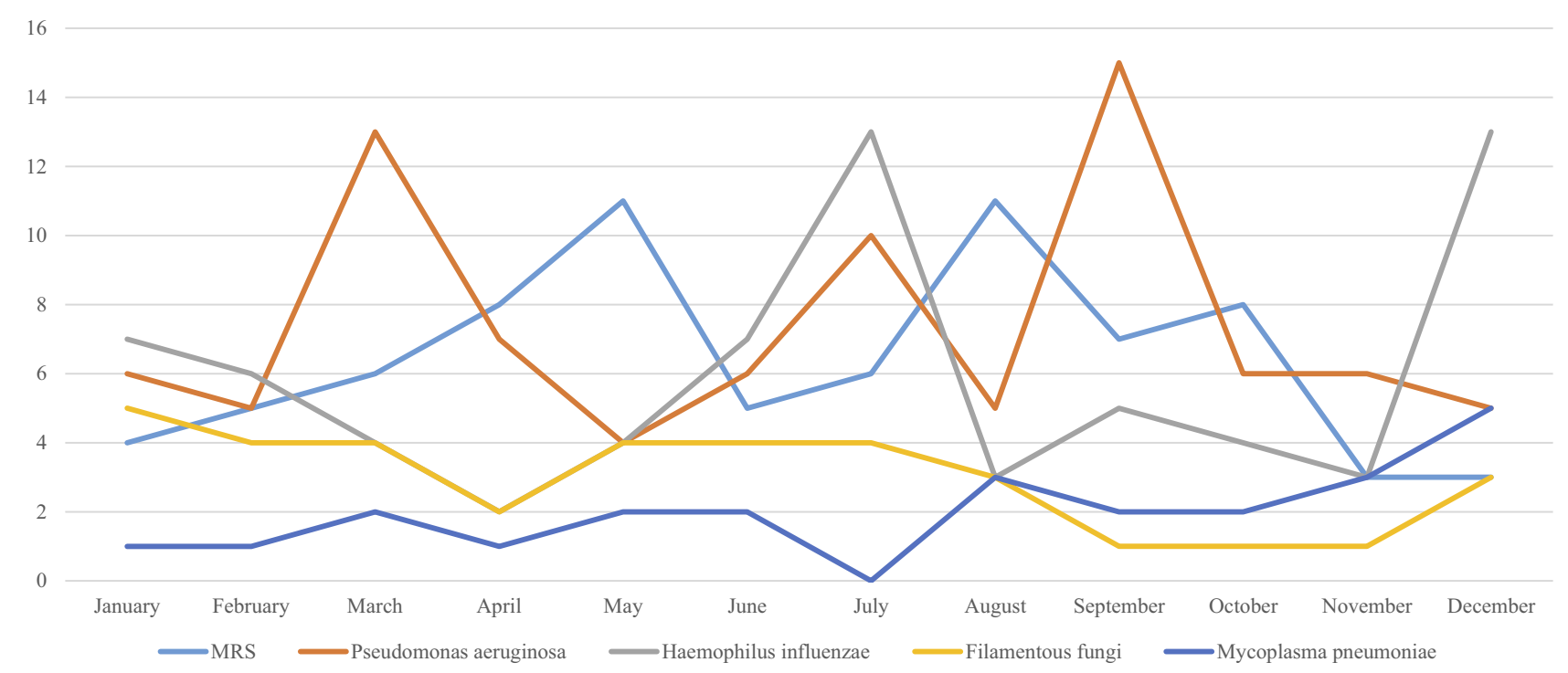

Figure 5 Seasonal distribution of predominant pathogens among overall CAP patients between September 2018 and August 2019.

Abbreviation: CAP, community-acquired pneumonia.

prevalent agents in patients with and without cancers, respectively.

The results of the univariate and multivariate logistic regression analysis revealed that in CAP patients without cancers, bronchiectasis was a risk factor for $P$. aeruginosa. Patients without other chronic diseases seemed to be more frequently affected by $M$. pneumoniae and showed fever (Table 4). CNS diseases and connective tissue diseases were associated with infections by MRS and $H$. influenzae, respectively (Tables 4 and 5). No risk factors were related to the predominant pathogens for CAP patients with cancers (Supplementary Table 3). There were also no risk factors associated with $K$. pneumoniae and $S$. pneumoniae for overall CAP patients (Supplementary Table 4).

\section{Discussion}

The number of cases of CAP in China ranged from 29.8 to 221.0 per 10,000 admissions. ${ }^{16}$ In our hospital, there were a total of 9274 CAP patients requiring hospitalization from September 2018 and August 2019. The patients were older with more coexisting medical conditions and approximately $5 \%$ of cases of CAP could be precisely treated for the detected pathogens. Because the research center was a tertiary medical care. With large improvements in the level of primary medical care and government efforts to promote primary medical insurance policies, ${ }^{17,18}$ young patients with CAP could be successfully treated in primary hospitals or cured by empirically oral antibiotics in outpatient settings. However, the elderly, who were easily exposed to bacterial colonization and difficult to be treated for its complexity with a variety of comorbidities (such as chronic diseases and cancer), needed more careful care in tertiary hospitals. Some patients were seriously ill and we could not obtain qualified sputum specimens for pathogens detection. In addition, some other pneumonias showed a dry cough, so there was no sputum for pathogen detection.

The results of our study showed that the number of cases of $P$. aeruginosa rose sharply in March and September. The CAP patients with cancers, who were infected with $K$. pneumoniae and $S$. pneumoniae were increasing in September and October, respectively. The northwest region of China has a warm season (March-May) and a cold season (September-November). Invasive pneumococcal disease commonly follows a seasonal pattern. ${ }^{19}$ Particularly, during seasonal changes from warm to cold weather, people who do not adapt to the season in a timely manner are susceptible to respiratory infections. Patients with cancers were more likely to be infected in a cold season. Surprisingly, the circulation of $H$. influenzae was highest in July in both the groups, which is inconsistent with literatures. ${ }^{20}$ More samples are needed to further study the phenomenon.

Compared with patients without cancers, we found that $77.18 \%$ of the CAP patients with cancers did not show typically clinical symptoms of fever. Besides, the median value of neutrophil, as one of the inflammatory indicators, was lower for cancer patients. In two previous studies, patients with postobstructive pneumonia due to malignancy were less likely to have leukocytosis, and serum inflammatory markers were significantly lower. ${ }^{21,22}$ Contributory mechanisms might include humoral immune deficit, cellular immune deficit or neutrophil 
Table I Characteristics of CAP Patients with and Without Cancers $(n=417)$

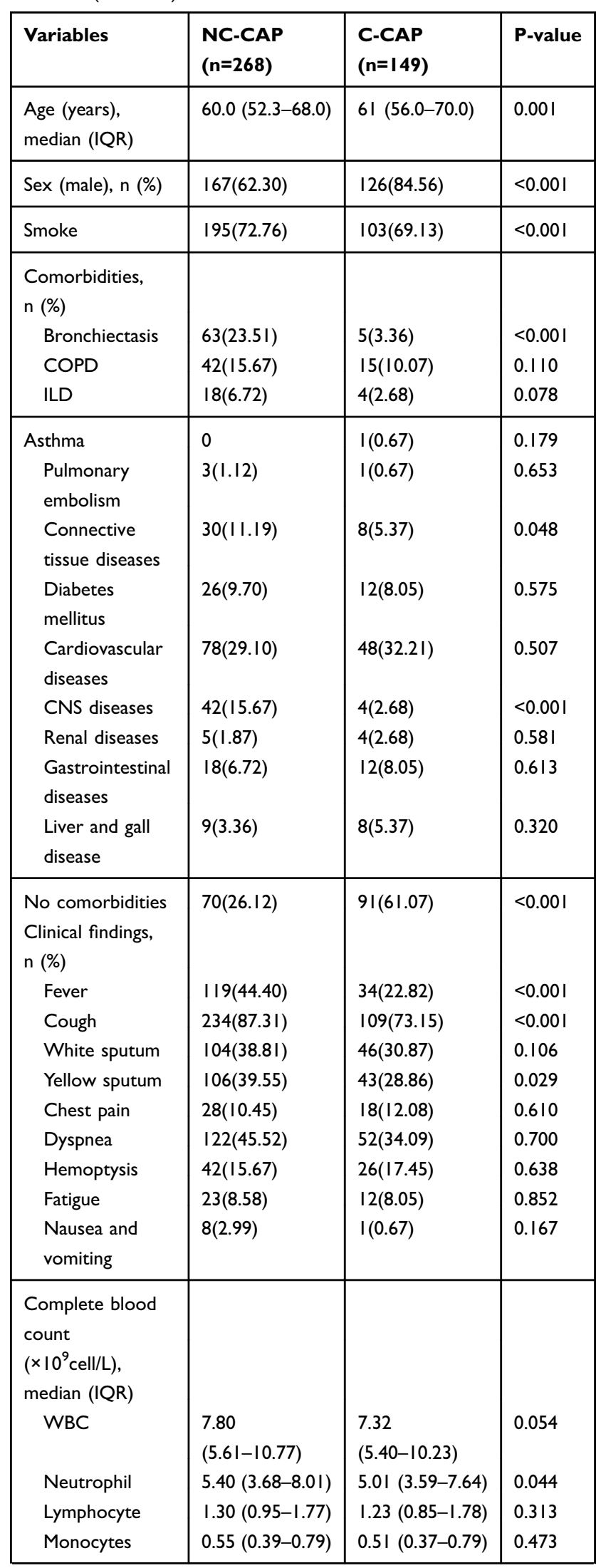

(Continued)
Table I (Continued).

\begin{tabular}{|c|c|c|c|}
\hline Variables & $\begin{array}{l}\text { NC-CAP } \\
(n=268)\end{array}$ & $\begin{array}{l}\text { C-CAP } \\
(n=149)\end{array}$ & P-value \\
\hline Platelet & $\begin{array}{l}236.00 \\
(172.00-295.75)\end{array}$ & $\begin{array}{l}231.00 \\
(173.00-307.00)\end{array}$ & 0.845 \\
\hline $\begin{array}{l}\text { CRP }(m g / L) \\
\text { Known, n (\%) } \\
\text { CRP > normal } \\
\text { value, n (\%) }\end{array}$ & $\begin{array}{l}128(47.76) \\
93(34.70)\end{array}$ & $\begin{array}{l}58(38.93) \\
39(26.17)\end{array}$ & $\begin{array}{l}0.082 \\
0.073\end{array}$ \\
\hline $\begin{array}{l}\text { PCT }(\mathrm{ng} / \mathrm{dL}) \\
\quad \text { Known, } \mathrm{n}(\%) \\
\mathrm{PCT}>0.5, \mathrm{n}(\%) \\
\mathrm{PCT}>0.5, \\
\text { median }(\mathrm{IQR})\end{array}$ & $\begin{array}{l}163(60.82) \\
14(5.22) \\
1.02(0.73-7.10)\end{array}$ & $\begin{array}{l}80(53.69) \\
14(9.40) \\
1.53(0.78-3.24)\end{array}$ & $\begin{array}{l}0.157 \\
0.103 \\
0.053\end{array}$ \\
\hline $\begin{array}{l}\text { Respiratory } \\
\text { failure, } n \text { (\%) }\end{array}$ & $60(22.39)$ & $16(10.74)$ & 0.003 \\
\hline $\begin{array}{l}\text { Body temperature } \\
\left({ }^{\circ} \mathrm{C}\right), \text { median } \\
(\mathrm{IQR})\end{array}$ & $37.0(36.7-38.7)$ & $36.8(36.5-37.2)$ & $<0.001$ \\
\hline
\end{tabular}

Abbreviations: SD, standard deviation; IOR, interquartile range; BALF, bronchoalveolar lavage fluid; COPD, chronic obstructive pulmonary disease; ILD, interstitial lung disease; CNS, central nervous system; WBC, white blood cell; CRP, C-reaction protein; PCT, procalcitonin; IQR, interquartile range; NC-CAP, no cancercommunity-acquired pneumonia; C-CAP, cancer-community-acquired pneumonia.

disorders. Moreover, cancer patients may experience neutropenia as a side effect of chemotherapy and radiotherapy.

It was reported that cancer patients with CAP had worse outcomes. ${ }^{23}$ Loss of $\mathrm{p} 53$, which is a common feature of human cancer, can aggravate lung injury during pneumonia. ${ }^{24}$ However, our population of CAP patients with cancer did not show worse respiratory failures. This could be attributed to the fact that patients selected for this study had relatively milder infections. Patients with severe infections, who were mechanically ventilated prior to specimen collection or had poor-quality lower respiratory tract sputum for pathogen detection, were excluded from the study.

We found that most of the samples in our study were BALF, which were better for the isolation of pathogens. The predominant pathogens were $H$. influenzae, M. pneumoniae, and S. pneumoniae, which were similar to those mentioned in the guidelines. ${ }^{15} \mathrm{H}$. influenzae was found to be significantly abundant in patients with cancer. P. aeruginosa and filamentous fungi, which were usually found in the nosocomial pathogen spectrum, were predominant in the group of patients without cancers. Because $73.88 \%$ of the patients without cancers suffered from chronic illnesses, and some cases received glucocorticoids treatment. 
Table 2 Etiologic Microorganisms of CAP Patients with and Without Cancers $(n=417)$

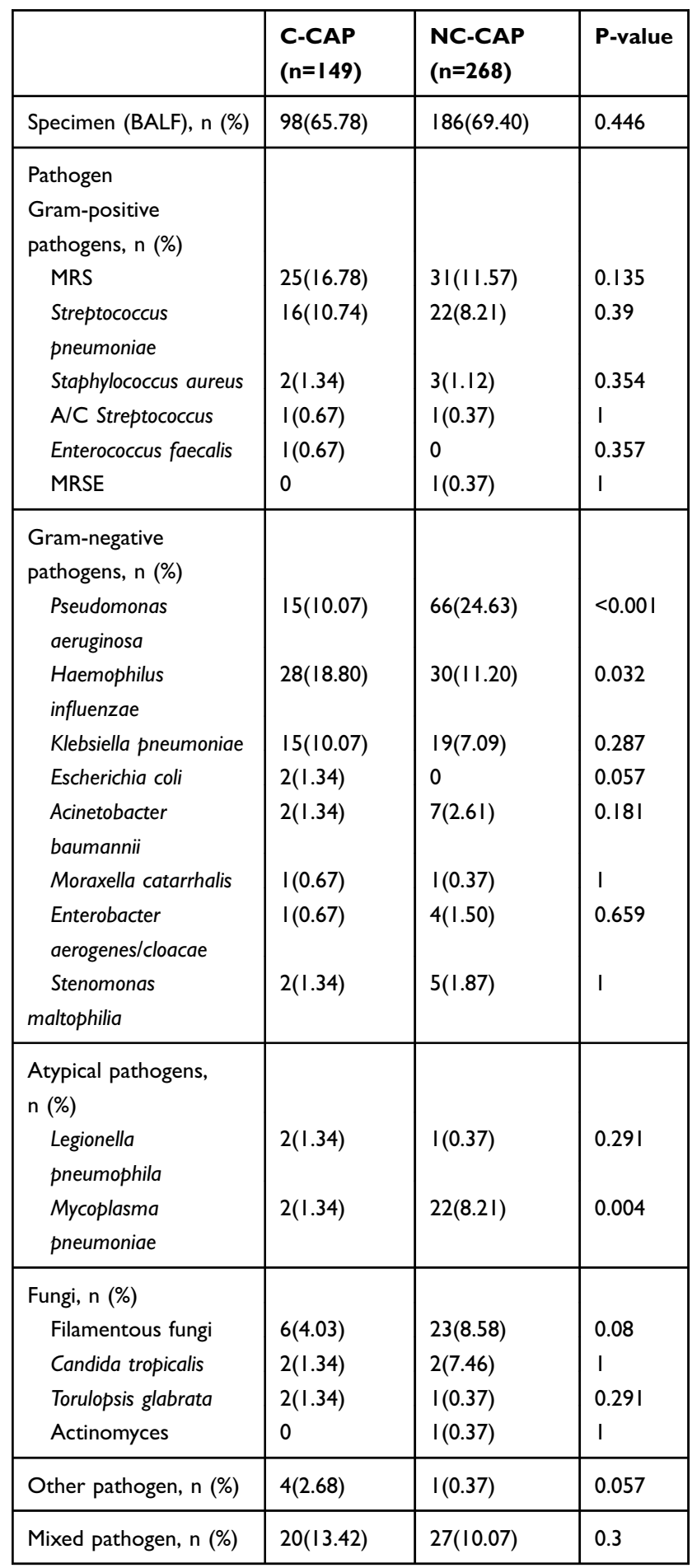

Abbreviations: MRS, methicillin-resistant Staphylococci; BALF, bronchoalveolar lavage fluid; MRSE, methicillin-resistant Staphylococcus epidermidis; NC-CAP, no cancercommunity-acquired pneumonia; C-CAP, cancer-community-acquired pneumonia.

MRS, which is a common colonization of the respiratory tract, consists mainly of Methicillin-resistant coagulase-negative staphylococci (MR-CoNS) and methicillin-resistant Staphylococcus aureus (MRSA). MR-
Table 3 Overall Distribution of Pathogens as Isolated Alone and/ or in Combination with Other Pathogens (Mixed Infection)

\begin{tabular}{|l|l|l|l|}
\hline & $\begin{array}{l}\text { C-CAP } \\
\text { (n=149) }\end{array}$ & $\begin{array}{l}\text { NC-CAP } \\
\text { (n=268) }\end{array}$ & P-value \\
\hline $\begin{array}{l}\text { Specimen (BALF), } \\
\mathrm{n}(\%)\end{array}$ & $98(65.78)$ & $186(69.40)$ & 0.953 \\
\hline $\begin{array}{l}\text { Pathogen } \\
\text { MRS }\end{array}$ & $35(23.49)$ & $42(15.68)$ & 0.049 \\
$\begin{array}{l}\text { Streptococcus } \\
\text { pneumoniae } \\
\text { Pseudomonas } \\
\text { aeruginosa } \\
\text { Haemophilus } \\
\text { influenzae } \\
\text { Klebsiella } \\
\text { pneumoniae } \\
\begin{array}{l}\text { Mycoplasma } \\
\text { pneumoniae } \\
\text { Filamentous fungi }\end{array}\end{array}$ & $24(16.11)$ & $31(11.57)$ & 0.189 \\
\hline
\end{tabular}

Abbreviations: MRS, methicillin-resistant Staphylococcus; NC-CAP, no cancercommunity-acquired pneumonia; C-CAP, cancer-community-acquired pneumonia.

CoNS is less pathogenic. But MRSA is generally regarded as one of the causative pathogens of nosocomial infections. It accounts for $20-40 \%$ of HAP cases and is globally limited in cases of CAP. ${ }^{25}$ It was estimated to occur in 0.51-0.64 cases per 100,000 inhabitants with communityacquired MRSA (CA-MRSA) pneumonia. ${ }^{26}$ One study in the USA showed that the rate of CA-MRSA pneumonia was $2.5 \%{ }^{27}$ In China, only a few cases of MRSA pneumonia were reported in CAP adolescents. ${ }^{15}$ However, MRS pneumonia cases were widely prevalent in our study. $S$. aureus was a frequent cause of pulmonary infection targeting individuals with underlying pulmonary conditions, including intubation, COPD, and especially cystic fibrosis. The independent risk factors for MRSA-CAP were reassessed as hospitalization for 2 days during the preceding 90 days, chronic dialysis during the preceding 30 days, congestive heart failure use of antibiotics in the previous 90 days, and use of gastric acid-suppressive agents. $^{28} 73.88 \%$ of our patients without cancers suffered from a variety of chronic diseases and needed frequent medical treatments. The immunosuppression was associated with all resistant pathogens including MRSA. Moreover, in some patients in the study, it was challenging to differentiate colonization from pathogens. The current guidelines for the treatment of pneumonia published by the American Thoracic Society and Infectious Diseases Society of America recommend empiric MRSA coverage 
Table 4 Univariate and Multivariate Logistic Regression Analyses of CAP Patients Without Cancers

\begin{tabular}{|c|c|c|c|c|c|c|}
\hline \multirow[t]{2}{*}{ Factors } & \multicolumn{2}{|c|}{ MRS OR(95\% Cl) } & \multicolumn{2}{|c|}{ Mycoplasma pneumoniae OR(95\% Cl) } & \multicolumn{2}{|c|}{ Pseudomonas aeruginosa $\mathrm{OR}(95 \% \mathrm{Cl})$} \\
\hline & Univariate & Multivariate & Univariate & Multivariate & Univariate & Multivariate \\
\hline Bronchiectasis & $\begin{array}{l}0.298 \\
(0.102-0.871)\end{array}$ & $0.258(0.084-0.794)$ & & & $\begin{array}{l}8.470 \\
(4.509-15.908)\end{array}$ & $\begin{array}{l}7.266 \\
(3.574-14.770)\end{array}$ \\
\hline CNS diseases & $\begin{array}{l}3.303 \\
(1.415-6.489)\end{array}$ & $2.511(1.130-5.580)$ & & & & \\
\hline $\begin{array}{l}\text { Connective tissue } \\
\text { diseases }\end{array}$ & & & & & & \\
\hline Sex & & & & & & \\
\hline Fever & & & $4.800(1.716-13.430)$ & $5.265(1.759-15.763)$ & & \\
\hline No comorbidities & & $0.360(0.146-0.888)$ & $7.442(2.890-19.164)$ & $4.093(1.511-11.086)$ & $0.316(0.148-0.677)$ & \\
\hline
\end{tabular}

Note: Blank cells indicate no statistical significance.

Abbreviations: CNS, central nervous system; OR, odds ratio; $\mathrm{Cl}$, confidence interval.

in at-risk patients, associated with significant mortality and morbidity. ${ }^{29}$ Finally, the apparent increase of MRS has resulted from improved techniques for the isolation of MRS. Newer polymerase chain reaction (PCR)-based molecular diagnostics for pathogen detection of the mecA gene, which is the genetic mechanism of resistance in community-acquired MRSA (CA-MRS) were used to detect methicillin-resistant staphylococci rapidly and sensitively. Methicillin resistance in $S$. aureus is primarily mediated by mecA, which is detected in our study by loopmediated isothermal amplification. However, mecA is also widely distributed among coagulase-negative staphylococci (CoNS). Thus, mecA alone cannot discriminate between MRSA and MR-CoNS. ${ }^{30}$ Also, the mecA- negative remnants of the SCC mec element may cause false-positive results. ${ }^{31}$ Inevitably, some patients may be mistakenly and empirically diagnosed as having MRSA infection.

The results of the univariate and multivariate logistic regression analysis indicated that the risk factor of bronchiectasis for CAP patients without cancers was associated with $P$. aeruginosa. M. Pneumoniae was prevalent in patients without any underlying diseases and showed the typical clinical manifestation of fever. Empirical therapy should include typical pathogen coverage for the patients. In addition, if CAP patients suffered from chronic CNS diseases, MRSA should be considered in the empirical therapy. Particular attention should be paid to

Table 5 Univariate and Multivariate Logistic Regression Analyses of CAP Patients Without Cancers

\begin{tabular}{|l|l|l|l|l|}
\hline \multirow{2}{*}{ Factors } & \multicolumn{2}{|l|}{ Filamentous Fungi OR(C195\%) } & \multicolumn{2}{l|}{ Haemophilus Influenzae } \\
\cline { 2 - 5 } & Univariate & Multivariate & Univariate & Multivariate \\
\hline Bronchiectasis & & & & \\
\hline CNS diseases & & & & $2.521(1.030-6.171)$ \\
\hline Connective tissue diseases & & & & $3.116(1.147-8.464)$ \\
\hline Sex & & & & \\
\hline Fever & & & & \\
\hline No comorbidities & & & & \\
\hline
\end{tabular}

Note: Blank cells indicate no statistical significance.

Abbreviations: CNS, central nervous system; OR, odds ratio; $\mathrm{Cl}$, confidence interval; CAP, community-acquired pneumonia. 
H. influenzae for CAP patients admitted with connective tissue diseases. No risk factors were found to be associated with CAP patients with cancer, for limited cases.

This study has some strengths and limitations. First, this is a single-center, retrospective, quasi-experimental design, which is not randomly assigned but it relies on documentation in electronic medical records for baseline characteristics. Critical patients who received mechanical ventilation and those that had been treated at secondary-care teaching hospitals were excluded from the study. It was difficult to distinguish CAP from VAP and HAP, so most of the selected patients had mild-moderate infection. Thus, the generalizability of our results was limited. Furthermore, a limited number of cases for some pathogens constrained the interpretation of seasonal trends.

\section{Conclusions}

In conclusion, lung cancer is one of the most important factors in patients with CAP. The CAP patients with cancers in our study were older and showed no typical clinical manifestations and changes in inflammatory markers. K. pneumoniae and $S$. pneumoniae were among the most commonly encountered pathogens in both the groups. $P$. pneumoniae, $M$. pneumoniae and filamentous fungi were predominant in CAP patients without cancers. $H$. influenzae and MRS were more prevalent for CAP cancer patients. Certain pathogens follow a seasonal pattern. In patients without cancers, MRS, $H$. influenzae and $P$. aeruginosa were associated with central nervous system (CNS) disease, connective tissue disease, bronchiectasis, respectively. In addition, healthy adults were likely to be infected with $M$. pneumoniae showing fever. Surprisingly, MRS was detected in our CAP patients with higher frequency than previously reported. Further studies are needed to support the findings.

The study provides suggestions for the empirical treatment of CAP patients in China with cancers and chronic diseases, who do not show typical clinical manifestations and laboratory results.

\section{Abbreviations}

CAP, community-acquired pneumonia; HAP, hospitalacquired pneumonia; VAP, ventilator-associated pneumonia; $\mathrm{SD}$, standard deviation; IQR, interquartile range; COPD, chronic obstructive pulmonary disease; ILD, interstitial lung disease; CNS, central nervous system; WBC, white blood cell; CRP, C-reaction protein; PCT, procalcitonin; NC-CAP, no cancer-community-acquired pneumonia; C-CAP, cancer- community-acquired pneumonia; MRSA, methicillinresistant Staphylococcus aureus; MRS, methicillin-resistant Staphylococci; M. pneumoniae, Mycoplasma pneumoniae; S. pneumoniae, Streptococcus pneumoniae; P. aeruginosa, Pseudomonas aeruginosa; H. influenzae, Haemophilus influenzae; K. pneumoniae, Klebsiella pneumoniae; MR-CoNS, Methicillin-resistant coagulase-negative staphylococci; MRSE, methicillin-resistant Staphylococcus epidermidis; CAMRSA, community-acquired MRSA; BALF, bronchoalveolar lavage fluid; LAMP, loop-mediated isothermal amplification; $\mathrm{OR}$, odds ratio; $\mathrm{CI}$, confidence interval.

\section{Ethical Approval}

This study was reviewed and approved by the Institutional Review Board of the Second Affiliated Hospital of Air Force Medical University. Informed consent was waived because the used data were fully anonymized and not traceable.

\section{Acknowledgments}

We thank Xiao-Qi Qiu for assistance with data collection. We would also like to thank Chen Li for providing statistical expertise with data analysis.

\section{Author Contributions}

All authors contributed to data analysis, drafting or revising the article, gave final approval of the version to be published, and agree to be accountable for all aspects of the work.

\section{Funding}

This research did not receive any specific grant from funding agencies in the public, commercial, or not-forprofit sectors.

\section{Disclosure}

The authors declare that they have no conflicts of interest.

\section{References}

1. World health organization. The top 10 causes of death. Available from: https://www.who.int/news-room/fact-sheets/detail/the-top-10-causesof-death. Accessed May 24, 2018.

2. Welte T, Torres A, Nathwani D. Clinical and economic burden of community-acquired pneumonia among adults in Europe. Thorax. 2012;67(1):71-79. doi:10.1136/thx.2009.129502

3. Pelton SI, Shea KM, Farkouh RA, et al. Rates of pneumonia among children and adults with chronic medical conditions in Germany. $B M C$ Infect Dis. 2015;15:470. doi:10.1186/s12879-015-1162-y

4. Hicks BM, Yin H, Bladou F, Ernst P, Azoulay L. Androgen deprivation therapy for prostate cancer and the risk of hospitalisation for community-acquired pneumonia. Thorax. 2017;72(7):596-597. doi:10.1136/thoraxjnl-2016-209512 
5. Schmedt N, Heuer OD, Häckl D, Sato R, Theilacker C. Burden of community-acquired pneumonia, predisposing factors and health-care related costs in patients with cancer. BMC Health Serv Res. 2019;19 (1):30. doi:10.1186/s12913-018-3861-8

6. Rolston K, Nesher L. Post-obstructive pneumonia in patients with cancer: a review. Infect Dis Ther. 2018;7(1):29-38. doi:10.1007/ s40121-018-0185-2

7. Nie XM, Li YS, Yang ZW, et al. Initial empiric antibiotic therapy for community-acquired pneumonia in Chinese hospitals. Clin Microbiol Infect. 2018;24(6):658.e1-658.e6. doi:10.1016/j.cmi.2017.09.014

8. Shinohara S, Sugaya M, Onitsuka T, Machida K, Matsuo M, Tanaka F. Impact of postoperative pneumonia developing after discharge on long-term follow-up for resected lung cancer. World J Surg. 2018;42(12):3979-3987. doi:10.1007/s00268-018-4727-2

9. Gowing SD, Chow SC, Cools-Lartigue JJ, et al. Gram-negative pneumonia augments non-small cell lung cancer metastasis through host toll-like receptor 4 activation. J Thorac Oncol. 2019;14 (12):2097-2108. doi:10.1016/j.jtho.2019.07.023

10. Musher DM, Thorner AR. Community-acquired pneumonia. $N$ Engl $J$ Med. 2014;371(17):1619-1628. doi:10.1056/NEJMra1312885

11. Gajdács M, Urbán E. Epidemiological trends and resistance associated with stenotrophomonas maltophilia bacteremia: a 10-year retrospective cohort study in a tertiary-care hospital in hungary. Diseases. 2019;7:2. doi:10.3390/diseases7020041

12. Restrepo MI, Mortensen EM, Velez JA, Frei C, Anzueto A. A comparative study of community-acquired pneumonia patients admitted to the ward and the ICU. Chest. 2008;133(3):610-617. doi:10.1378/chest.07-1456

13. Cillóniz C, Ewig S, Polverino E, et al. Microbial aetiology of community-acquired pneumonia and its relation to severity. Thorax. 2011;66(4):340-346. doi:10.1136/thx.2010.143982

14. Gajdács M, Urbán E. Prevalence and antibiotic resistance of stenotrophomonas maltophilia in respiratory tract samples: a 10-year epidemiological snapshot. Health Serv Res Manag Epidemiol. 2019;6:2333392819870774. doi:10.1177/2333392819870774

15. Cao B, Huang Y, She DY, et al. Diagnosis and treatment of community-acquired pneumonia in adults: 2016 clinical practice guidelines by the Chinese thoracic society, Chinese medical association. Clin Respir J. 2018;12(4):1320-1360. doi:10.1111/ crj. 12674

16. Zhu YG, Tang XD, Lu YT, Zhang J, Qu JM. Contemporary situation of community-acquired pneumonia in China: a systematic review. $J$ Transl Int Med. 2018;6(1):26-31. doi:10.2478/jtim-2018-0006

17. Wang S, Xu J, Jiang X, et al. Trends in health resource disparities in primary health care institutions in Liaoning Province in Northeast China. Int J Equity Health. 2018;17(1):178. doi:10.1186/s12939-0180896-8

18. The State Council of the People's Republic of China. The 13th five-year plan for deepening reform of the medical and health care systems, Beijing 2017. Available from: http://www.gov.cn/zhengce/ content/2017-01/09/content 5158053.htm.
19. Dowell SF, Whitney CG, Wright C, Rose CE Jr, Schuchat A. Seasonal patterns of invasive pneumococcal disease. Emerg Infect Dis. 2003;9(5):573-579. doi:10.3201/eid0905.020556

20. Rubach MP, Bender JM, Mottice S, et al. Increasing incidence of invasive haemophilus influenzae disease in adults, Utah, USA. Emerg Infect Dis. 2011;17(9):1645-1650. doi:10.3201/eid1709.101991

21. Abers MS, Sandvall BP, Sampath R, et al. Postobstructive pneumonia: an underdescribed syndrome. Clin Infect Dis. 2016;62 (8):957-961. doi:10.1093/cid/civ1212

22. Urbaniak A, Zięba M, Zwolińska A, et al. Comparison of local and systemic inflammatory markers in patients with community-acquired pneumonia and pneumonia coexisting with lung cancer. Pneumonol Alergol Pol. 2011;79(2):90-98.

23. José R, Mohammed AO, Goldring J, Chambers RC, Brown JS, Agarwal B. Cancer patients with community-acquired pneumonia treated in intensive care have poorer outcomes associated with increased illness severity and septic shock at admission to intensive care: a retrospective cohort study. Pneumonia (Nathan). 2015;6:77-82. doi:10.15172/pneu.2015.6/645

24. Madenspacher JH, Azzam KM, Gowdy KM, et al. p53 Integrates host defense and cell fate during bacterial pneumonia. J Exp Med. 2013;210(5):891-904. doi:10.1084/jem.20121674

25. Gajdács M. The continuing threat of methicillin-resistant Staphylococcus aureus. Antibiotics (Basel). 2019;8:2. doi:10.3390/ antibiotics 8020052

26. Vardakas KZ, Matthaiou DK, Falagas ME. Incidence, characteristics and outcomes of patients with severe community acquired-MRSA pneumonia. Eur Respir J. 2009;34(5):1148-1158. doi:10.1183/ 09031936.00041009

27. Dunaway S, Orwig KW, Arbogast ZQ, Myers ZL, Sizemore JA, Giancola SE. Evaluation of a pharmacy-driven methicillin-resistant Staphylococcus aureus surveillance protocol in pneumonia. Int J Clin Pharm. 2018;40(3):526-532. doi:10.1007/s11096-018-0647-3

28. Shindo Y, Ito R, Kobayashi D, et al. Risk factors for drug-resistant pathogens in community-acquired and healthcare-associated pneumonia. Am J Respir Crit Care Med. 2013;188(8):985-995. doi:10.1164/rccm.201301-0079OC

29. Metlay JP, Waterer GW, Long AC, et al. Diagnosis and treatment of adults with community-acquired pneumonia. an official clinical practice guideline of the American thoracic society and infectious diseases society of America. Am J Respir Crit Care Med. 2019;200(7): e45-45e67. doi:10.1164/rccm.201908-1581ST

30. Chen C, Zhao Q, Guo J, Li Y, Chen Q. Identification of Methicillin-Resistant Staphylococcus aureus (MRSA) using simultaneous detection of mecA, nuc, and femB by Loop-Mediated Isothermal Amplification (LAMP). Curr Microbiol. 2017;74 (8):965-971. doi:10.1007/s00284-017-1274-2

31. Becker K, Denis O, Roisin S, et al. Detection of mecA- and mecC-positive Methicillin-Resistant Staphylococcus aureus (MRSA) Isolates by the new Xpert MRSA Gen 3 PCR assay. J Clin Microbiol. 2016;54(1):180-184. doi:10.1128/JCM.02081-15
Infection and Drug Resistance

\section{Publish your work in this journal}

Infection and Drug Resistance is an international, peer-reviewed openaccess journal that focuses on the optimal treatment of infection (bacterial, fungal and viral) and the development and institution of preventive strategies to minimize the development and spread of resistance. The journal is specifically concerned with the epidemiology of antibiotic resistance and the mechanisms of resistance development and diffusion in both hospitals and the community. The manuscript management system is completely online and includes a very quick and fair peerreview system, which is all easy to use. Visit http://www.dovepress.com/ testimonials.php to read real quotes from published authors. 